[0212-7199 (2006) 23: 4; pp 153-155] ANALES DE MEDICINA INTERNA Copyright () 2006 ARAN EDICIONES, S.L.

AN. MED. INTERnA (Madrid) Vol. 23, N. ${ }^{\circ}$, pp. $153-155,2006$

\section{Prevalencia de la mutación C677T del gen de la metilentetrahidrofolato reductasa en pacientes con patología isquémica cerebrovascular aguda en la Comunidad Autónoma Aragonesa}

\author{
B. SÁNCHEZ-MARÍN, J. M. GRASA ${ }^{1,4}$, M. TORRES ${ }^{1}$, M. T. CALVO ${ }^{2}$, \\ B. MARTÍNEZ-JARRETA ${ }^{3}$, J. A. GARCÍA-ERCE ${ }^{1}$, M. GIRALT ${ }^{1}$
}

Servicios de Neurología, 'Hematología y Hemoterapia y ${ }^{2}$ Genética. Hospital Universitario Miguel Servet. ${ }^{3}$ Laboratorio de Genética Forense. Departamento de Medicina Legal y Toxicología. ${ }^{4}$ Departamento de Microbiología, Epidemiología y Salud Pública. Universidad de Zaragoza

\title{
RESUMEN
}

Fundamentos: La mutación C677T de la metilentetrahidrofolato reductasa (MTHFR) es la principal causa de hiperhomocisteinemia moderada en nuestro medio. La hiperhomocisteinemia es un factor reconocido de riesgo para aterotrombosis. Los pacientes con la mutación MTHFR C677T padecen hiperhomocisteinemia moderada en situaciones de carencia de folatos.

Métodos: Se revisaron retrospectivamente resultados de los estudios de la mutación C677T MTHFR en pacientes con accidentes cerebrovasculares isquémicos agudos (ACVA) menores de 50 años y mayores de 50 años sin factores clásicos de riesgo vascular o historia familiar o personal sugerente de trombofilia, en un periodo de 3 años. Se realizaron estudios de la misma mutación en 90 donantes de sangre voluntarios sanos, como grupo control. Se realizó estadística descriptiva en base de datos informatizada.

Resultados: Se recogieron muestras de 99 pacientes y de 90 controles. Edad media: 44,3 con desviación estándar de 13,9 años en pacientes y 39,1 con DS de 8,3 años en controles. Encontramos $19(19,19 \%)$ homocigosis MTHFR C677T en el grupo de pacientes y $14(15,55 \%)$ en el grupo de controles .

Conclusiones: La homocigosis MTHFR C667T es más frecuente en el grupo de pacientes con ACVA que en los controles, si bien no encontramos diferencias significativas. Sin embargo, sugerimos que, dada la alta prevalencia poblacional encontrada en nuestro medio para la mutación MTHFR C677T, su estudio debe realizarse dentro de los estudios de trombofilia, pues es capaz de identificar pacientes con un factor de riesgo reversible mediante la administración de folatos.

PALABRAS CLAVE: Homocisteína. Ictus. Trombofilia. MTHFR. Folatos.

Sánchez-Marín B, Grasa JM, Torres M, Calvo MT, Martínez-Jarreta B, García-Erce JA, Giralt M. Prevalencia de la mutación C677T del gen de la metilentetrahidrofolato reductasa en pacientes con patología isquémica cerebrovascular aguda en la Comunidad Autónoma Aragonesa. An Med Interna (Madrid) 2006; 23: 153-155.

\section{INTRODUCCIÓN}

Se han descrito la importancia de la hiperhomocisteinemia como factor de riesgo vascular en general y de accidente cere-

Este trabajo es el resultado del proyecto presentado en el VIII Curso de Enfermedades Cerebrovasculares para Médicos Residentes de Neurología. Ha sido financiado parcialmente por la beca CONSID PO49/99.
PREVALENCE OF METHYLENETETRAHYDROFOLATE REDUCTASE C677T MUTATION AMONG PATIENTS WITH ACUTE ISCHEMIC CEREBROVASCULAR DISEASE IN ARAGON

\section{ABSTRACT}

Background: Mutation C677T of the methylenetetrahydrofolate reductase (MTHFR) is the main cause of mild hyperhomocysteinemia. Hyperhomocysteinemia is a recognized risk factor for aterothrombosis. MTHFR C677T patients have higher levels of homocysteine in absence of dietary folates.

Methods: Retrospective study over data from patients studied for MTHFR C677T diagnosed of ischemic stroke (IS) younger 50 or older 50 without classic vascular risk factors or with familiar or personal history suggesting thrombophilia in a period of 3 years. MTHFR C677T was screened in 90 healthy blood donors as a control group. Computer database was used for descriptive statistics.

Results: Blood simples from 99 patients and from 90 donors (control). Mean age: 44.3 with Standard desviation (SD) 13.9 years in IS group and 39.1 with SD 8.3 years in control group. We found 19 (19.19\%) homozygotes for MTHFR C677T in IS group and $14(15.55 \%)$ in control group.

Conclusions: Homozygosis for MTHFR C667T is more frequent in the IS group than in the control one, although there is no significant differences. Anyway, we suggest that, because of the high prevalence of the mutation MTHFR C677T found, screening should be made in the thombophilia studies, so that we could find patients with a risk factor that could be lowered by folates in the diet.

KEY WORDS: Homocystein. Stroke. Thrombofilia. MTHFR. Folate. brovascular agudo en particular (1-10). La etiología de la hiperhomocisteinemia es muy variada, provocándose niveles altos de homocisteina en sangre en la insuficiencia renal crónica, insuficiencia hepática, desnutrición, toma de anticonceptivos orales, mutaciones en la Cistation beta sintetasa, antiepilépticos, Ldopa y fármacos que interfieren las vías metabólicas de los folatos, tabaquismo, etc. (11-15). Incluso se han observado diferencias en función del sexo, siendo mayor la homocisteinemia en 
varones (16-18). No obstante, en la población general, es mucho más frecuente encontrar sujetos con homocisteina débil o moderadamente elevada, sin padecer ninguna de las circunstancias ya descritas. La principal causa de hiperhomocisteinemia moderada en nuestro medio es una mutación en la posición 677 de gen de la 5,10-metilentetrahidrofolato redutasa (MTHFR), que consiste en un cambio de citosina $(\mathrm{C})$ por timidina $(\mathrm{T})$ a nivel genómico y por consiguiente un cambio de alanina por valina a nivel proteico (19). El $73 \%$ de pacientes con homocisteína superior a 40 $\mathrm{mcmol} / \mathrm{L}$ son homocigotos para C677T MTHFR (20). La prevalencia de homocigotos para esta mutación en población europea oscila entre el 5 y el 15\%. La mutación C677T de la MTHFR provoca una variedad termolábil de dicha enzima que, en los sujetos homocigotos (genotipo TT), muestra una reducción cercana al $50 \%$ en la capacidad de donar grupos metilo a la enzima metionina sintetasa (21), con lo que disminuye la velocidad de conversión de homocisteína hacia metionina, elevándose los niveles de homocisteína.

El estudio que presentamos pretende determinar la prevalencia del alelo $\mathrm{T}$ en posición $677 \mathrm{del}$ gen de la MTHFR en pacientes con ACVA jóvenes o mayores de 50 años con ausencia de factores de riesgo clásicos, y en un grupo de donantes de sangre sanos.

\section{MATERIAL Y MÉTODOS}

Pacientes. Se realizó estudio genético para la mutación C677T MTHFR en pacientes diagnosticados de ACVA que se refirireron para estudio de trombofilia hereditaria y adquirida, dentro de la rutina diagnóstica habitual del hospital terciario que atiende las áreas 2 y 5 de salud de la comunidad autónoma aragonesa. Rutinariamente se refieren a estudio de trombofilia los pacientes con ACVA menores de 50 años y mayores de 50 años sin factores de riesgo vascular (tabaquismo, hipertensión arterial, arritmias embolígenas, diabetes mellitus, hiperlipemia). Retrospectivamente, y a partir de los registros de los estudios de de trombofilia hereditaria y adquirida desde octubre de 1998 a mayo de 2001, se revisaron las historias clínicas de los pacientes enviados a estudio con diagnóstico confirmado de ACVA de manera consecutiva en el periodo de tiempo referido, y se analizaron edad y sexo.

Controles. Se realizó estudio en donantes de sangre voluntarios y altruistas consecutivos que acudieron al banco hospitalario de sangre, previo consentimiento informado enviado al comité de ética del hospital.
Recogida de especímenes. Se recogieron muestras de sangre en tubos que contenían EDTA tripotásico para extracción de ADN por medios convencionales, al menos tres meses tras el evento isquémico agudo en el caso de los pacientes, y en el momento de la donación altruista de sangre en el caso de los controles. Estos últimos rellenaron un cuestionario y un consentimiento informado.

Determinaciones laboratoriales. Se realizaron PCR-RFLP para detección de C677T MTHFR según procedimientos previamente descritos (19).

Se realizó estadística descriptiva en base de datos informatizada.

\section{RESULTADOS}

Se recogieron muestras de 99 pacientes (M/F: 44/55). La edad media fue de 44,3 años con una desviación estándar (DS) de 13,9 años (IC95\%: 16,5-72,1). Se recogieron muestras de 90 donantes altruistas de sangre (M/F: 51/39) con edad media de 39,1 años y DS de 8,3 años (IC95\%: 22,5-55,7).

En el grupo de pacientes encontramos 19 homocigosis TT (variedad mutante), 52 heterocigotos (CT) y 28 homocigotos CC (variedad silvestre). En el grupo de controles encontramos 14 homocigosis TT (variedad mutante), 43 heterocigotos (CT) y 33 homocigotos CC (variedad silvestre). En la tabla I se describen los datos epidemiológicos de ambos grupos.

\section{DISCUSIÓN}

Encontramos una elevada frecuencia alélica de la variedad mutada en pacientes $(0,45)$ con un $19,19 \%$ de homocigotos, si bien en donantes sanos la incidencia de homocigotos $(15,55 \%)$ y la frecuencia alélica $(0,39)$ también son elevadas. Parece que la frecuencia alélica es superior en el grupo de pacientes que en el de controles, pero la significación estadística no es valorable, pues al encontrar una prevalencia tan alta en población sana, el tamaño de la muestra necesario para establecer conclusiones es muy elevado, y excede las ambiciones de este estudio. Los resultados encontrados en la serie de controles hacen que estimemos una alta prevalencia poblacional del polimorfismo T en posición 677 del gen de la MTHFR en la población sometida a estudio. La prevalencia descrita se encuentra entre las más altas descritas en el continente europeo, sin embargo el territorio aragonés tiene una de las tasas más bajas de Europa en mortalidad cardiovascu-

\section{TABLA I}

RESULTADOS EPIDEMIOLÓGICOS DEL ESTUDIO

\begin{tabular}{lcccccc}
\hline & \multicolumn{3}{c}{ Pacientes } & \multicolumn{3}{c}{ Controles (\%) } \\
\hline $\mathrm{n}(\%)$ & $\mathrm{C} / \mathrm{T}$ & $\mathrm{C} / \mathrm{T}$ & $\mathrm{C} / \mathrm{C}$ & $\mathrm{T} / \mathrm{T}$ & $\mathrm{C} / \mathrm{T}$ & $\mathrm{C} / \mathrm{C}$ \\
Edad (años) & $19(19,2)$ & $52(52,5)$ & $28(28,3)$ & $14(15,5)$ & $43(47,7)$ & $33(36,6)$ \\
Sexo (M/F) & $47(\mathrm{DS}: 12,2)$ & $47,1(\mathrm{DS}: 13)$ & $40,4(\mathrm{DS}: 16,2)$ & $38(\mathrm{DS}: 9,4)$ & $39,5(\mathrm{DS}: 8,1)$ & $39(\mathrm{DS}: 8,1)$ \\
Frecuencia alelo T & $8 / 11$ & $24 / 28$ & $12 / 16$ & $8 / 6$ & $24 / 19$ & $19 / 14$ \\
\hline
\end{tabular}

T/T: Homocigotos para variedad mutante de la MTHFR; C/T: heterocigotos; C/C: homocigotos para la variedad silvestre o normal; DS: desviación estándar; $M$ : varones; $F$ : mujeres. 
lar. Probablemente las interacciones ambientales y lo que se denomina "dieta mediterránea" son capaces de modular la tendencia genética, más o menos intensa, que mutaciones de este tipo otorgan (22). La "americanización" de la dieta puede ser un factor determinante para que en muchos individuos el potencial aterogénico de C677T MTHFR se manifieste. Recordemos que muchos paises con patrones dietéticos pobres en aporte de verdura y fruta (y por tanto, deficitarios en folatos) han adoptado medidas como el enriquecimiento de productos básicos con folatos.

Hay que tener en cuenta que la MTHFR es una enzima dependiente de flavin-adenina dinucleótido (FAD) que cataliza la conversión de 5,10-metilenterahidrofolato en 5metiltetrahidrofolato de manera irreversible. La mutación C677T crea una variedad termolábil de la enzima con menor capacidad en la transferencia de un grupo metilo para la remetilación de la homocisteina para su conversión a metionina por medio de la enzima metionina sintetasa, que requiere vitamina B12 (cobalamina) como cofactor (12). Diversos trabajos sugieren que la actividad de MTHFR en la mayoría de los tejidos alcanza niveles suficientes para el normal metabolismo en pacientes con un adecuado aporte de folatos en la dieta $(23,24)$. Como consecuencia de ello, en pacientes con genotipo TT, los niveles de homocisteina disminuyen con aportes diarios en la dieta de 0,5 a $2 \mathrm{mg}$ de ácido fólico (25).

Es difícil llegar a conclusiones claras con respecto a esta mutación dada la gran cantidad de factores dietéticos que pueden influir en la expresión de la hiperhomocisteinemia provocada o favorecida por dicha mutación (26). Es muy posible que la variabilidad de conclusiones a las que están llegando numerosos estudios sobre la MTHFR C677T como factor de riesgo independiente de enfermedad aterotrombótica exprese el diferente aporte en la dieta de folatos y vitaminas de grupo B según la localización geográfica y aspectos socioculturales. No obstante, el conocimiento de este aspecto genético en un individuo que, sin factores de riesgo clásicos, desarrolla ACVA, puede aportar posibilidades profilácticas con folatos sobre un "nuevo" factor de riesgo, la homocisteína.

\section{Bibliografía}

1. Perry IJ, Refsum H, Morris RW, Ebrahim SB, Ueland PM, Shaper AG. Prospective study of serum total homocysteine concentration and risk of stroke in middle-aged British men. Lancet 1995; 346: 1395-8.

2. Zheng YZ, Tong J, Do XP, Pu XQ, Zhou BT. Prevalence of methylenetetrahydrofolate reductase $\mathrm{C} 677 \mathrm{~T}$ and its association with arterial and venous thrombosis in the Chinese population. Br J Haematol 2000; 109: 870-4.

3. Ueland PM, Refsum H, Beresford SA, Vollset SE. The controversy over homocysteine and cardiovascular risk. Am J Clin Nutr 2000; 72: 324-332.

4. Madonna P, de Stefano V, Coppola A, Cirillo F, Cerbone AM, Orefice $\mathrm{G}$, et al. Hyperhomocysteinemia and other inherited prothrombotic conditions in young adults with a history of ischemic stroke. Stroke 2002; 33: $51-6$

5. González Ordóñez AJ, Medina Rodríguez JM, Fernández Álvarez CR, Sánchez García J, Fernández Carreira JM, Álvarez Martínez MV, et al. Lowering high levels of fasting total homocysteine with folic acid and vitamins B in patients with venous thromboembolism: relationship between response and the C677T methylenetetrahydrofolate reductase (MTHFR) genotype. Med Clin (Barc) 2000; 114: 7-12.

6. Nowak-Göttl U, Strater R, Heinecke A, Junker R, Koch HG, Schuierer $\mathrm{G}$, et al. Lipoprotein (a) and genetic polymorphisms of clotting factor $\mathrm{V}$, prothrombin and methylenetetrahydrofolate reductase are risk factors of spontaneous ischemic stroke in childhood. Blood 1999; 94: 3678-82

7. Kohara K, Fujisawa M, Ando F, Tabara Y, Niino N, Miki T, et al. MTHFR gene polymorphism as a risk factor for silent brain infarcts and white matter lesions in the japanese general population. Stroke 2003; 34: 1130-1135.

8. Yoo JH, Choi GD, Kang SS. Pathogenicity of thermolabile methylenetetrahydrofolate reductase for vascular dementia. Arterioscler Thromb Vasc Biol 2000; 20: 1921-5.

9. Eikelboom JW, Hankey GJ, Anand SS, Lofthouse E, Staples N, Baker RI. Association between high homocyst(e)ine and ischemic stroke due to large- and small-artery disease but not other etiologic subtypes of ischemic stroke. Stroke 2000; 31: 1069-75.

10. Cardo E, Monros E, Colome C, Artuch R, Campistol J, Pineda M, et al. Children with stroke: polymorphism of the MTHFR gene, mild hyperhomocysteinemia, and vitamin status. J Child Neurol 2000; 15: 295-8.

11. Jacobsen DW. Homocysteine and vitamins in cardiovascular disease. Clin Chem 1998; 44: 1833-1843.

12. Ueland PM, Hustad S, Schneede J, Refsum H, Vollset SE. Biological and clinical implications of the MTHFR C677T polymorphism. Trends Pharmacol Sci 2001; 22: 195-201

13. Smulders YM, de Man Am, Stehouwer CD, Slaats EH. Trimethoprim and fasting plasma homocysteine. Lancet 1998; 352: 1827-1828.

14. Yoo JH, Hong SB. A common mutation in the methylenetetrahydrofola- te reductase gene is a determinant of hyperhomocysteinemia in epileptic patients receiving anticonvulsivants. Metabolism 1999; 48: 1047-1051.

15. Ordóñez LA, Wurtman RJ. Folic acid deficiency and methyl group metabolism in rat brain: effects of L-dopa. Arch Biochem Biophys 1974; 160: 372-376.

16. Dierkes J, Jeckel A, Ambrosch A, Westphal S, Luley C, Boeing H. Factors explaining the difference of total homocysteine between men and women in the European Investigation Into Cancer and Nutrition Postdam study. Metabolism 2001; 50: 640-645.

17. Dekou V, Whincup P, Papacosta O, Ebrahim S, Lennon L, Ueland PM, et al. The effect of the C677T and A1298C polymorphisms in the methylenetetrahydrofolate reductase gene on homocysteine levels in elderly men and women from the British regional heart study. Atherosclerosis 2001; 154: 659-66.

18. Wu Y, Tomon M, Sumino K. Methylenetetrahydrofolate reductase gene polymorphism and ischemic stroke: sex difference in Japanese. Kobe J Med Sci 2001; 47: 255-62.

19. Frosst P, Blom HJ, Milos R, Goyette P, Sheppard CA, Matthews RG, et al. A candidate genetic risk factor for vascular disease: a common mutation in the methylenetetrahydrofolate reductase. Nat Genet 1995; 10: 111-113.

20. Guttormsen AB, Ueland PM, Nesthus I, Nygard O, Schneede J, Vollset $\mathrm{SE}$, et al. Dterminants ans vitamin responsiveness of intermediate hyperhomocysteinemia $(<$ or $=40 \mathrm{micromol} / \mathrm{liter})$. The Hordaland Homocysteine Study. J Clin Invest 1996; 98: 2174-2183.

21. Kang SS, Zhou J, Wong PWK, Kowalisyn J, Strokaosch G. Intermediate homocysteinemia: a thermolabile variant of the methylenetetrahydrofolate reductase. Am J Hum Genet 1988; 43: 414-421.

22. Grasa JM, Torres M, Sánchez-Marín B, García-Erce JA, Calvo MT, Giralt M. Hiperhomocisteinemia y mutación C677T de la metilentetrahidrofolato reductasa. An Med Interna (Madrid) 2002; 19: 269.

23. Stern LL, Bagley PJ, Rosenberg IH, Selhub J. Conversion of 5-formyltetrahydrofolic acid to 5-methyltetrahydrofolic acid is unimpaired in folateadequate persons homozygous for the C677T mutation in the methylenetetrahydrofolate reductase gene. J Nutr 2000; 130: 2238-2242.

24. Nixon PF, Bertino JR. Effective absorption and utilization of oral formyltetrahydrofolate in man. N Engl J Med 1972; 286: 175-179.

25. Malinow MR, Nieto FJ, Kruger WD, Duell PB, Hess DL, Gluckman RA, et al. The effects of folic acid supplementation on plasma total homocysteine are modulated by multivitamin use and methylenetetrahydrofolate reductase genotypes. Arterioscler Thromb Vasc Biol 1997; 17: 1157-1162.

26. Mayer O Jr, Äimon J, Bosolová H, Mromdka M, Äubrt I, Vobrubová J. The effects of folate supplementation on some coagulation parameters and oxidative status surrogates. Eur J Clin Pharmacol 2002; 58: 1-5. 